Anuario del Instituto de Historia Argentina, vol. 17, nº 1, e032, junio 2017.

ISSN 2314-257X

Universidad Nacional de La Plata.

Facultad de Humanidades y Ciencias de la Educación.

Centro de Historia Argentina y Americana

\title{
El mundo obrero latinoamericano ante la Organización Internacional del Trabajo a lo largo del siglo XX
}

\author{
Andrés Stagnaro *; Laura Caruso ** \\ * Instituto de Investigaciones em Humanidades y Ciencias Sociales - Universidad Nacional \\ de La Plata - CONICET; ** Instituto De Altos Estudios Sociales - Universidad Nacional de \\ San Martin - Universidad de Buenos Aires - CONICET, Argentina I \\ andres.stagnaro81@gmail.com; lauracaruso@gmail.com
}

A poco de cumplirse el aniversario centenario de la creación de la Organización Internacional del Trabajo (en adelante OIT), este actor es puesto en el centro de una renovada historiografía que se piensa en clave regional y que aborda de una forma crítica y novedosa la vinculación de este organismo internacional y América Latina.

Para quienes estamos interesados desde una perspectiva histórica en los mundos del trabajo latinoamericanos, sus formas, sujetos, experiencias, contornos y dinámicas, la OIT se torna un actor insoslayable. Desde su creación en 1919 constituye un factor fundamental para pensar tanto la construcción de la intervención de los estados nacionales en esos mundos laborales como, sobre todo, la definición de ciertos campos laborales constituidos en torno a aquello que la OIT problematizó y definió como trabajo, en función de un conjunto de sentidos y supuestos a los que es necesario historizar y repensar. Precisamente, la definición de tales campos laborales en el seno de la OIT estuvo atravesada por las tensiones entre los estados, los empresarios y los trabajadores, cuyas representaciones tenían cabida en este particular órgano internacional con una estructura tripartita.

En la construcción de diversos campos así entendidos (trabajo marítimo, trabajo infantil, trabajo femenino, trabajo en áreas no metropolitanas, trabajo indígena, trabajo forzado, por mencionar algunos) se expresaron las demandas y disputas de intereses y posiciones entre estos tres sujetos - empleadores, estados y trabajadores-, sus expectativas y estrategias, pero también aquellas tensiones entre los diversos países del concierto mundial y sus jerarquías, reproducidas al interior de tales discusiones, y expresadas en las 
definiciones de esos campos de trabajo.

Un consenso basal configuró desde su creación lo que fuera, tal vez, la mayor particularidad de la construcción ginebrina, y en el cual América Latina participó fuertemente: la noción de trabajo que definía un campo iba atada a una serie de derechos laborales, fundamentales, reconocidos y asumidos como necesidad en el contexto de la primera posguerra y de un mundo convulsionado por la guerra y la revolución, donde la paz, pero sobre todo la justicia social -ambos principios sostenidos como objetivos primordiales de la organización - garantizaban el proyecto integrador que la OIT venía a desplegar.

Desde América Latina, indagar este proceso es necesariamente, creemos, reponer la centralidad de la propia región en y con la OIT, destacando esa dimensión vincular, en donde esa mutua relación es parte constitutiva de ambos sujetos. Los estudios específicos de esta relación, hasta el momento, pusieron el acento en las relaciones de los diversos países latinoamericanos con el organismo. Esta centralidad es consecuencia directa de las existencias de las fuentes y de los requerimientos propios de la construcción de un campo académico aun tímidamente explorado. Aun así es posible percibir ciertos comportamientos y patrones en la relación de estos países que son comunes. Por caso la designación de Argentina como país miembro del governing body -el órgano ejecutivo de la asamblea de la OIT- en la primera conferencia de Washington fue celebrado como una conquista de toda la región por el resto de los delegados latinoamericanos. Fue común también que los distintos países latinoamericanos respondieran de forma similar a los estímulos legislativos de la OIT. Incluso con diferentes ritmos, en la mayoría de los casos quedaba en evidencia la tensión entre ser considerados países en condición especial, en tanto su desarrollo industrial estaba aun alejado de los patrones de los países centrales, para quienes la legislación propuesta era aun muy avanzada -y por tanto eso disminuía su valía en el concierto de naciones-, o estimar posible la chance de incorporar las convenciones aun cuando la realidad económica interna no estuviese preparada. Esta tensión fue evidente a lo largo de los primeros años de la OIT, y reactualizada en diversas ocasiones, cada vez que un debate sobre las convenciones volvía a poner en evidencia el reflejo de la distancia que separa a la región de los países centrales. Cuestiones como el trabajo indígena desde la década del sesenta del siglo pasado, o el trabajo forzado aun en la actualidad constituyen momentos claves para comprender esta relación conflictiva entre las pretensiones de modernidad de la región y su consideración como zona especial. Algunos aportes recientes sobre este tema están siendo conceptualizados desde la región, como por caso la idea de pensar esta relación en torno a una geografía diferencial de derechos para pensar el vínculo de la OIT con América Latina, desarrollada por Rossana Barragán.

También es posible observar una preocupación compartida por los países de la región y derivada de esta consideración como países en situación especial o no, que podríamos denominar -parafraseando a los estudiosos del problema indígena- los riesgos del ventrilocuismo en la relación entre América Latina y la OIT. Producto mismo de las relaciones de poder al interior del organismo, la preeminencia de las delegaciones de los países centrales en los debates, y hasta el origen nacional de los funcionarios de la institución ginebrina, conllevaban un riesgo temido por los latinoamericanos de que sus problemas fueran pronunciados en su nombre.

Por último la acumulación de trabajos sobre la OIT y distintos países de la región permite al menos esbozar una cronología mínima, parcial e inestable, plausible de ser modificada a partir de nuevos trabajos empíricos, de la relación entre la OIT y América Latina. Esta cronología es también con y desde América Latina. Sin dudas el desarrollo de la propia OIT con sus vaivenes impactó en la temporalidad propuesta, en tanto también es una construcción relacional. Por caso, si bien sobrevivió al colapso de la Sociedad de Naciones, el traslado de su sede durante la Segunda Guerra Mundial y su virtual refundación por medio de la declaración de Filadelfia en 1944 constituye un parteaguas fundamental para el organismo; sin embargo, el impacto de esta medida en la región fue posterior. 
A grandes rasgos podemos identificar un primer momento, entre 1919 y 1925, donde la relación entre la región latinoamericana y la OIT fue de grandes expectativas. En este período, dicha relación estuvo marcada por una distancia expectante, producto en gran parte de cierta desconfianza sobre el carácter universal de la OIT. No era la primera vez que se intentaba constituir un organismo dedicada a promover la legislación del trabajo a escala planetaria, y sus antecedentes no habían logrado trascender de forma permanente las fronteras impuestas por el propio desarrollo industrial. Entre 1925 y 1936 atendemos a los intentos institucionalizadores como el signo de una nueva etapa. Superadas las suspicacias iniciales, los países latinoamericanos comienzan a tejer lazos de carácter permanente con la OIT. Se establece así una red de corresponsales y se consolidan los ya estrechos vínculos académicos. Además la OIT comienza a percibir en América Latina un terreno fecundo para su prédica centrada en la paz y la justicia social. Esto se traduce en las visitas que realizara su director, Albert Thomas, a distintos países de la región en 1925. A partir de 1936 y hasta mediados de la década del cincuenta un nuevo periodo puede concebirse como caracterizado por cierta autonomía de los países de la región en relación a la OIT, favoreciendo el desarrollo de una agenda propia al interior del organismo. La primera conferencia del trabajo regional de los estados miembros de la OIT $-\mathrm{y}$ además la primera de su tipo- se desarrolló en la ciudad de Santiago de Chile en 1936 y a partir de allí se realizaron con continuidad hasta el día de hoy -incluso cuando la propia OIT debió suspender sus reuniones anuales en razón del desarrollo de la guerra ente 1939 y 1944-. Esta reunión no solo marcó los comienzos de una agenda presentada por los propios países participantes latinoamericanos, sino también dio cuenta de la madurez de las relaciones entre la OIT y la región. Su presencia ya no es puesta en entredicho, y por el contrario, realza los vínculos entre los países de la región en su seno. A las reuniones de los estados miembros le siguió la conformación de una oficina permanente para la región en la ciudad de Lima aun hoy en funciones. Desde mediados de la década del cincuenta y hasta fines de los sesenta hay una pérdida de importancia relativa de América Latina en su relación con la OIT, que coincide con un proceso de descentralización propuesto desde el mismo organismo. Los procesos de descolonización de África y parte de Asia conllevan la atención del organismo. Sin embargo los vínculos establecidos en los años previos permitieron la continuidad de la agenda propia, y las misiones a algunos países volvieron a rescatar por momentos el vínculo privilegiado entre la OIT y la región, pero ahora en forma bilateral con algunos de sus estados miembros. Este período estuvo marcado también por los resquemores que en algunos actores despertaba una institución que no dejaba de ser percibida como imperialista en el marco de la guerra fría. Desde fines de los sesenta y hasta mediados de los ochenta el ascenso de regímenes autoritarios y dictaduras militares volvieron a tensionar la relación entre la región y la OIT en puntos centrales como la libertad sindical o la persecución a los líderes sindicales. La década del ochenta da comienzo a una nueva etapa en donde el foco estará puesto en lograr la consolidación de las democracias emergentes, pero al mismo tiempo la preocupación central estará en las dificultades para lograr los objetivos del organismo en torno a la situación laboral a la vista de las recurrentes crisis económicas -desde la crisis de la deuda mexicana que dio origen a la década perdida-. Con la aplicación de las reformas neoliberales comienza la última etapa. En este periodo la preocupación del organismo se plasmó en la consigna-objetivo trabajo decente, justo cuando la región ingresa en un tobogán de precarización y terciarización del trabajo.

Los trabajos que aquí presentamos son nuevos aportes en esta búsqueda colectiva en la que varios de los autores venimos trabajando desde hace ya un tiempo y es la propuesta de la Red Latinoamericana de Estudios sobre la OIT, la que impulsamos desde el año 2015. Por este motivo las monografías que se publican en el presente dossier adquieren aun mayor relevancia cuando son complementadas y puestas en debates con las publicaciones que ya hemos producido desde la red y con los trabajos que varios colegas vienen desarrollando para comprender los vínculos de la OIT con América Latina.

Presentados en torno a un orden cronológico, este conjunto de artículos muestra la potencia y la necesidad del diálogo regional, de la construcción dialogada y colaborativa de conocimiento, eje de la Red y del oficio. 
En primer lugar, Guihlerme Nunes en su escrito "Ócio e lazer na regulamentação das férias operárias: a OIT e o caso brasileiro na primeira metade do século XX" rastrea el surgimiento del derecho a las vacaciones pagas para la clase asalariada en el seno de la OIT y en casos de la región latinoamericana, donde Argentina aparece como una fuerte referencia. Toma en particular los años 20 como momento de desarrollo primordial de la temática, y en el caso particular de Brasil, al cual se aboca, como coyuntura donde se originan las primeras leyes al respecto, y rol de las demandas y acciones obreras en su constitución. La observación atinada que realiza el autor sobre el despliegue renovado de la lucha por las vacaciones entre los gremios en Brasil luego de sancionada la primera ley, en 1925, repone el interés por pensar la relación entre la consolidación de derechos y los nuevos horizontes de la protesta obrera. Una nueva ley en 1934 le permite indagar cuánto de las críticas y reivindicaciones surgidas de la primera ley y expresadas en diversos conflictos se plasmó en la nueva legislación, y qué vinculación tuvieron ambas con el planteamiento ginebrino del problema.

Nuestro propio trabajo, "Representantes y representaciones de Argentina en la Organización Internacional del Trabajo en la década de 1920”, aborda una cuestión central para la ideología de la OIT: las tensiones que derivan en la elección de los delegados que representarían al país y garantizarían la representación tripartita. A partir de los informes de estos delegados nos propusimos tejer los vínculos que estos elaboraron entre sí como representantes argentinos- como con otros delegados, fundamentalmente trabajadores, que les permitieron formar solidaridades más allá de las fronteras nacionales. La posibilidad de presentar una delegación tripartita, permite reponer también la tensión de ser considerad un país especial o como parte de las naciones con mayor desarrollo industrial. El especial foco en los delegados sindicales apunta a visibilizar este actor central en la legitimidad ginebrina y en la participación argentina, cuya designación fue foco de tensiones y negociaciones.

En su artículo "Pelos pequenos trabalhadores do Novo Mundo: OIT e trabalho infantojuvenil nas Américas (1936-1939)”, José Pacheco analiza los debates y nociones en las conferencias regionales del trabajo de América Latina realizadas en Chile (1936) y Cuba (1939) para pensar desde allí a la región con una voz propia y no como mera apropiación de los convenios y debates ginebrinos. De manera lúcida, el autor encuentra en esa voz propia, más que la comprobación de una realidad laboral sobre la cual legislar, la construcción performativa de infancias y juventudes modélicas, en tanto agentes del futuro o trabajadores del mañana, y el rol de la familia como contexto laboral legítimo. Rastrea a la vez las formas en que la construcción de una regulación internacional sobre el trabajo de niños, niñas y jóvenes a través de la confección de convenios, y, lo que se torna central, su ratificación y debate por los diversos países.

Por su parte Juan Carlos Yañez, a esta altura un pionero y especialista en los estudios de la OIT, analiza en "La Organización Internacional del Trabajo y la libertad sindical en América Latina: el caso de Venezuela en 1949” los vínculos del sindicalismo latinoamericanista con la OIT a partir de la misión de asistencia técnica enviada por el organismo al país caribeño en 1949. El estudio pormenorizado de las fuentes le permite no solo dar cuenta de los mecanismos desplegados en estas misiones, sino también las complejidades para enfrentarse a actores cuyas lógicas de intervención estaban cruzadas por su condición de clase, pero también por un profundo nacionalismo que se interponía como una barrera difícil de superar para las pretensiones universalizantes de la OIT. Además la situación específica de Venezuela -bajo un gobierno dictatorial y que no había ratificado, y por tanto otorgado carácter legal, a la convención No87 sobre libertad sindical y derecho a la sindicalización-planteo a la OIT desafíos novedosos sobre cómo lograr cumplir que sus estados miembros cumpliesen con las convenciones, sin transformarse en una suerte de legislatura universal que horadase las soberanías nacionales.

Por otro lado, Luciana Zorzoli en su trabajo "La OIT y las dictaduras latinoamericanas: una aproximación al Caso 842 contra Argentina” precisamente aborda las denuncias contra la política sindical de la última 
dictadura argentina ante la OIT, y la forma en que ese espacio internacional se tornó un área por momentos de legitimación, en otros de disputa y negociación, de la dirigencia sindical con el gobierno militar. Ambos sujetos, sindicalistas y gobierno dictatorial, cambiaron de estrategias en el período que va desde la presentación del caso, en 1976 a la crítica coyuntura de 1979. La autora reconstruye, de manera precisa y en base a una novedosa documentación que vale la pena destacar, los contenidos y formas de esas estrategias, sus construcciones argumentativas y tensiones, así como sus cambios.

Por último, cierra este dossier el trabajo de Victoria Basualdo "La OIT entre la dictadura y la democracia en la Argentina: aportes sobre el papel de organizaciones internacionales en la reconfiguración de las relaciones laborales en la primera mitad de los años 80". En un exhaustivo trabajo de fuentes la autora nos presenta la intrincada red de relaciones que se tejieron a partir de la OIT entre el movimiento obrero argentino y algunas organizaciones sindicales de fuerte incidencia a nivel mundial, como la Confederación Internacional de Organizaciones Sindicales Libres (CIOLS), y con presencia en el seno del organismo. Esta red fue fundamental en la elaboración de las denuncias internacionales contra la dictadura argentina -por ejemplo el caso 842 analizado por Luciana Zorzoli- y habilitó un espacio de suma importancia en la defensa de los derechos delos trabajadores en un momento de suma regresión de los mismos en un contexto de desarticulación social y económica, pero al mismo tiempo se presentó como un actor conservador en el contexto inmediatamente posterior con el retorno de la democracia.

En su conjunto los trabajos presentados dan continuidad a la tarea planteada, en la reconstrucción monográfica de diferentes períodos de la relación de la OIT con los países de América Latina. Aún mediante sus conclusiones parciales y particulares, habilitan la proyección de estudios más generales que favorezcan la comprensión de esta relación en sus múltiples escalas y en la diversidad de sus actores involucrados. 